\title{
DC MOTOR CONTROL SYSTEM THROUGH ANDROID APPLICATION USING ARDUINO NANO
}

\author{
A.C. GHEORGHE ${ }^{1}$, M. I. MATEI ${ }^{2}$ \\ Valahia University of Targoviste, Faculty of Electrical Engineering, Electronics and Information Technology \\ E-mail: gheorghe_andrei89@yahoo.com
}

\begin{abstract}
The study aims for the development of a control system for D.C motors through an application made for Android mobile devices. The D.C motors are represented by a robot model car and the control application communicates with the system via Bluetooth technology. The Android mobile device user must install an application on his mobile device, then, the user must turn on the Bluetooth communication on the mobile device. The user can use various commands to control the robot model car such as front, back, stop, left, right. These commands are sent from the Android mobile device to the Bluetooth receiver, which is interfaced with the control system. The control system has a Bluetooth HC-06 receiver that receives commands from the control application and sends them to the Arduino Nano microcontroller to control the motors through two L298N drivers.
\end{abstract}

Keywords: D.C Motor, Bluetooth, Control, Arduino, Android

\section{INTRODUCTION}

Nowadays, mobile devices come equipped with highperformance processors, larger storage capacities and have more communication abilities. The smartphone, is a small device that has the processing power to do all kind of things and is changing the ways that humans interact with machines. Smartphones gradually became a universal portable device and gave people a daily use for different tasks. In the past years, the Android OS (Operating System) has gained more and more popularity in smartphones. The Android platform comes with a full software package, this software package is made of an OS, a middleware layer and basic applications. The difference from other existing platforms, like the iPhone OS, is that it comes with a software used as a development kit (SDK) [1].

The D.C motor is mostly used for different drive applications in the industrial field, the mai applications are in automation, traction, civil and military systems because of its efficiency, silent operation, small size, reliability, and low maintenance [2].

Because of the progress in the wireless technology field, several communication methods such as Bluetooth and Wi-Fi are introduced. Each connection has a certain distinctive description and utilization. Among the Wi-Fi connection, Bluetooth technology is mostly used. Bluetooth is mostly used for data transfer and adds new characteristics to smartphones. The Bluetooth technology was invented by Ericsson in the year 1994 and has shown a big advantage by adding this technology in smartphones. The communication method changed the way users utilize their mobile devices at home/office, and it has also helped with the transition of wired devices to wireless devices. A Bluetooth device that acts like a host has the ability to communicate with maximum seven Bluetooth devices in the same time via a single connection. Communication is very important in everyday life and the Bluetooth technology is useful in a control system as means of control [3]. The Bluetooth communication method is used to control the movement direction of the motors. The controlled system is represented by a small robot model car that consists of an Arduino Nano microcontroller connected with a Bluetooth HC-06 module, two L298N driver modules and four D.C motors. When the Android application will be connected to this system via a Bluetooth connection, the user can manipulate the small robot model car through wireless commands given in the application. The robot model car can move left, right, forward and backward.

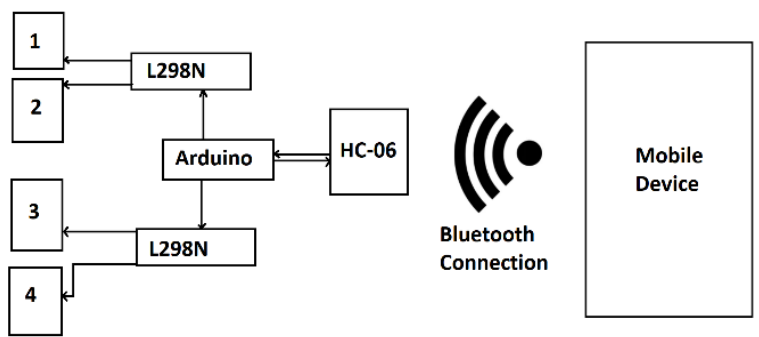

Figure 1. System block diagram.

In Figure 1 we have the block diagram of the system. The controlled system receives the users command from the mobile devices via a Bluetooth connection through the HC-06 Bluetooth module, then the Arduino Nano processes the command received and operates the two L298N drivers that are used to select the speed and the direction of the four D.C motors.

\subsection{Arduino Nano}

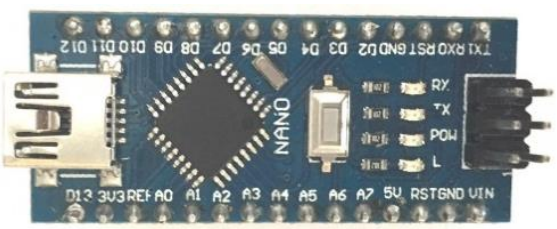

Figure 2. Arduino Nano developing board. 
The Arduino Nano development board (e.g. Figure 2) is a small development board measuring $18 \times 45 \mathrm{~mm}$. The development board uses the ATmega328 microcontroller, this microcontroller has a modified 8-bit AVR Atmel processor running at a frequency of $16 \mathrm{MHz}$.[4] The development board has $32 \mathrm{~KB}$ of FLASH type memory, 2 $\mathrm{KB}$ of SRAM type memory, $1 \mathrm{~KB}$ of EEPROM type memory, 6 analog pins (A0-A5) and 14 digital pins (of which 6 pins support a PWM type output). The development board operates at a voltage of $5 \mathrm{~V}$ and provides a current of $40 \mathrm{~mA}$ on the output/input pins (50 $\mathrm{mA}$ on the $3.3 \mathrm{~V}$ output pin). The communication protocols supported by the Arduino Nano development board are I2C, SPI, USART [5]. The Android Nano technical specifications are:

- Microcontroller: Atmega328

- Working voltage: $5 \mathrm{~V}$

- $\quad$ Recommended input voltage: 7V-12V

- Limit input voltage: 6V-12V

- Digital pins: 14 (6 PWM output)

- Analog pins: 6

- Current per I / O pin: $40 \mathrm{~mA}$

- $\quad 3.3 \mathrm{~V}$ current: $50 \mathrm{~mA}$

- Flash memory: $32 \mathrm{~KB}$ of which $0.5 \mathrm{~KB}$ for bootloader

- $\quad$ SRAM: $2 \mathrm{~KB}$

- EEPROM: $1 \mathrm{~KB}$

- Clock speed: $16 \mathrm{MHz}$

\subsection{Bluetooth HC-06}

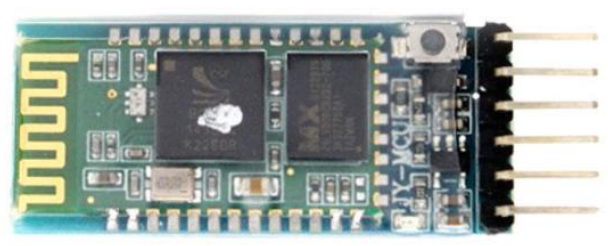

Figure 3. HC-06 Bluetooth module.

The HC-06 Bluetooth Module (e.g. Figure 3) is a Class 2 slave module that is made for wireless serial communications. If the module is paired with a primary Bluetooth device (e.g. computer, smartphone, tablet) its transmission becomes transparent to the user. All the data received by the module via the serial input from the microcontroller is immediately transmitted and when the module receives data, it is sent via the serial interface. Interfacing the module with a microcontroller doesn't need a specific software code because of the serial interface mention above. The HC-06 Bluetooth module operates at voltage between $3.6 \mathrm{~V}$ and $6 \mathrm{~V}$. The HC-06 technical specifications are:

- Bluetooth chip: CSR

- Technology: Bluetooth 2.0 + EDR

- Operating voltage: $3.6 \mathrm{~V}-6 \mathrm{~V}$

- UART interface with configurable baud rate

- Supports $8 \mathrm{Mb}$ external flash

- $\quad$ Supports 802.11 protocol

\subsection{L298N driver}

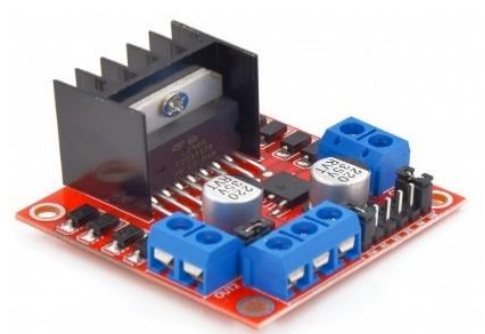

Figure 4. L298N motor driver.

The L298N module (e.g. Figure 4) is a powerful motor driver which is used for driving DC and stepper motors. The L298N module is used for controlling up to 4 D.C motor or for controlling the direction and speed of 2 D.C motors. The module is made from a IC L298, resistors and capacitors, $5 \mathrm{~V}$ voltage regulator, power supply indicator and a $5 \mathrm{~V}$ power jumper that is used for activating the voltage regulator when the jumper is inserted. If the user chooses to operate the driver with a power supply that is less or equal to $12 \mathrm{~V}$, then the module will be power by the voltage regulator and the $5 \mathrm{~V}$ pin can supply power to the microcontroller. The pins that are responsible for the motor speed are ENA and ENB, the motor speed is controlled by a PWM input signal. The pins that are responsible for the direction of both the motors are IN1, IN2, IN3 and IN4, the direction is controlled by applying a HIGH logic input signal. The L298N technical specifications are:

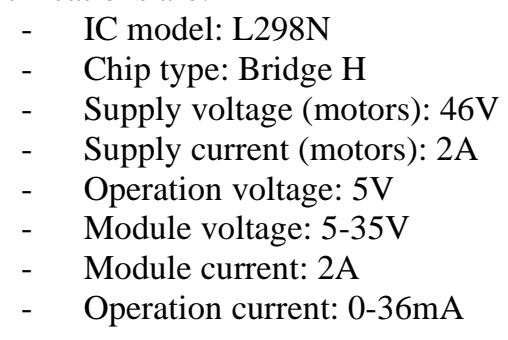

\subsection{DC motor with reducer}

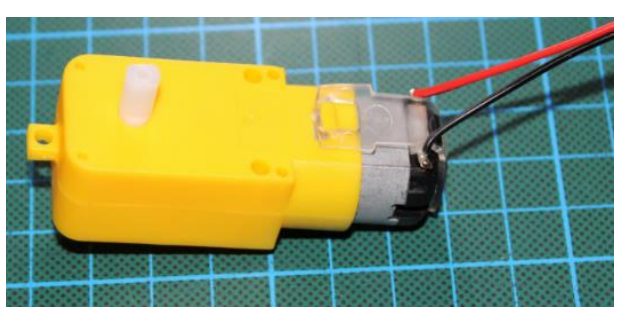

Figure 5. DC motor with reducer.

The D.C motor with reducer (e.g. Figure 5) uses a voltage between $3 \mathrm{~V}$ and $6 \mathrm{~V}$. The reducer ratio is $1: 48$ and at $3 \mathrm{~V}$ has 125 RPM with a current consumption of $60 \mathrm{~mA}$, at $5 \mathrm{~V}$ has $200 \mathrm{RPM}$ with a current consumption of $100 \mathrm{~mA}$ and at $6 \mathrm{~V}$ has $230 \mathrm{RPM}$ with a current consumption of 120 $\mathrm{mA}$. The D.C motor with reducer technical specifications are:

\footnotetext{
- Engine reduction: 1:48

- Motor supply voltage: 3V-6V DC

Torque: $0.8 \mathrm{~kg} * \mathrm{~cm}$
} 
RPM: 3V: $125 \mathrm{rpm}, 5 \mathrm{~V}: 200 \mathrm{rpm}, 6 \mathrm{~V}: 230 \mathrm{rpm}$ - Current: 3V: $60 \mathrm{~mA}, 5 \mathrm{~V}: 100 \mathrm{~mA}, 6 \mathrm{~V}: 120 \mathrm{~mA}$

\section{THE ELECTRONIC BOARD}

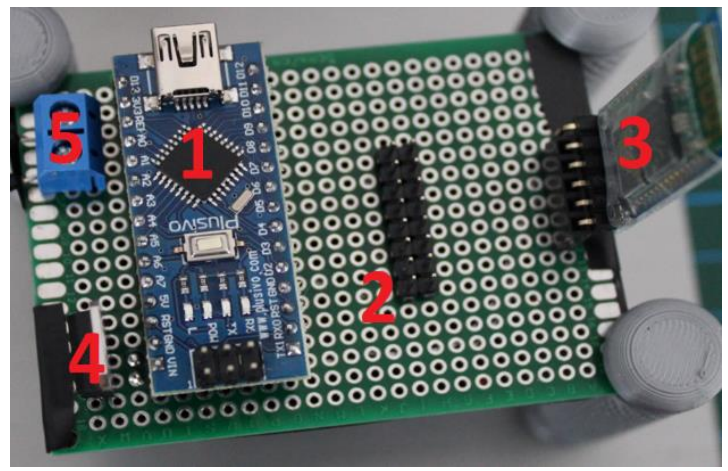

Figure 6. Electronic Board.

The electronic board (e.g. Figure 6) is made from the Arduino Nano developing board (1) that is used to control the motor driver, motor driver connectors (2) that are used to connect the L298N drivers, HC-06 Bluetooth module (3) that is use to send commands to the Arduino, L7805 voltage regulator (4) that is used to supply $5 \mathrm{~V}$ to the developing board and Bluetooth module and the power connector (5) that is used to supply power to the entire system.

\section{THE FUNCTIONING PRINCIPLE}

The system gives the user the ability to control the speed and the movement direction of four D.C motors that are represented by a small model car through an Android application that is developed for mobile devices. The user connects to the model car via a Bluetooth connection and sends commands for controlling the model car. The application sends a text message to the HC-06 slave module then the text message is processed by the Arduino Nano that controls the L298N drivers thus controlling the DC motors [6]. The text message " 1 " is used for forward movement, the text message " 2 " is used for backward movement, the text message " 3 " is used for left movement, the text message " 4 " is used for right movement and the text message " 0 " is use to stop the DC motors.

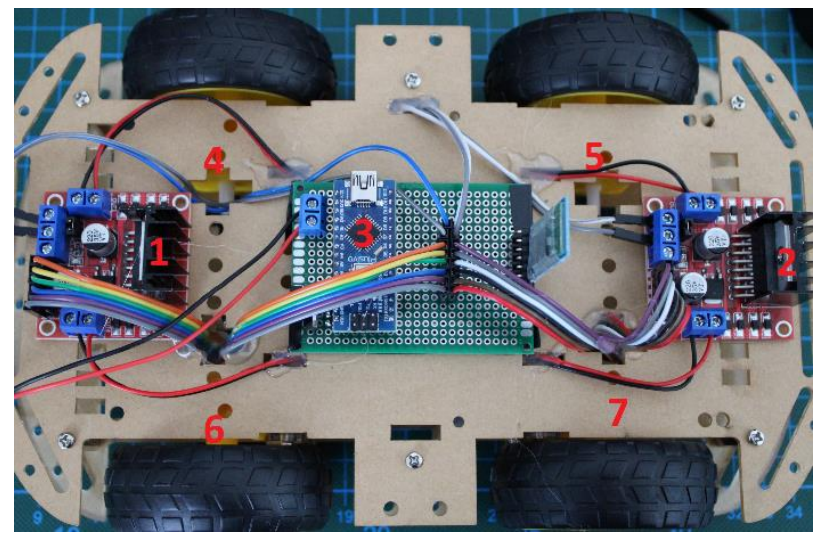

Figure 7. DC motor control system.
In Figure 8 we have the controlled model car fully assembled. The electronic board (3) is connected to the L298N drivers $(1,2)$ that are used to control the D.C motors $(4,5,6,7)$.

\subsection{Software application}

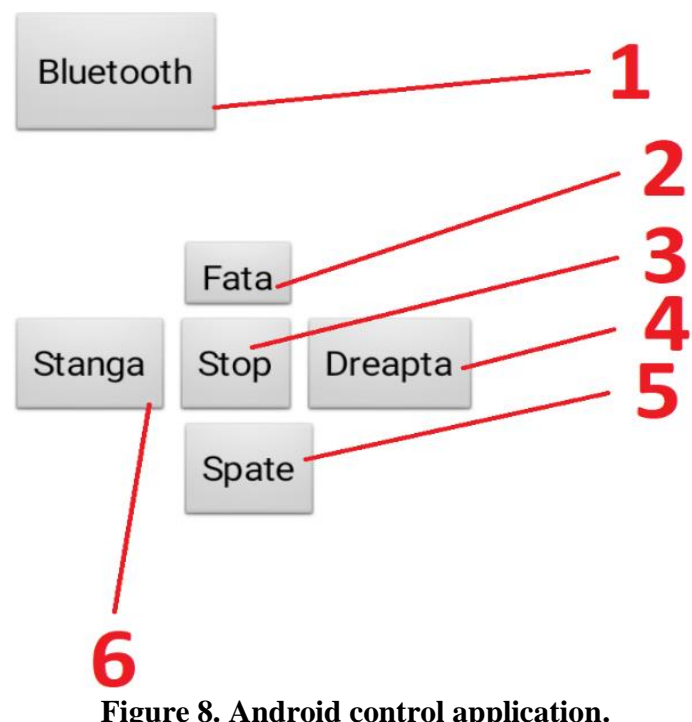

The Android control application (e.g. Figure 8) has 6 buttons. The Bluetooth button (1) is used to connect to the HC-06 slave module, the forward button (2) is used to send the text message " 1 ", the stop button (3) is used to send the text message " 0 ", the backward button (5) is used to send the text message " 2 ", the left button (6) is used to send the text message " 3 " and the right button (4) is used to send the text message " 4 ".

\section{CONCLUSIONS}

This paper is an introduction to the world of control systems using Bluetooth technology and smart mobile devices based on the Android operating system. The Android smartphone operating system is a suitable environment for developing an efficient and easy to customize program, which allows the user to control via a Bluetooth connection the created system. The controlled system uses the Atmega328 microcontroller, this microcontroller was intended for the realization of various technical solutions in the field of electronics, mechatronics and automation. Most wireless control systems use RF modules, but the paper demonstrates that the use of Bluetooth technology is a similar or better solution to effectively and securely control certain systems that use a wireless connection. The L298N module that controls the D.C motors is easy to implement together with a development board like the one used to create this system and the D.C motors with a small size / power still present a viable solution for various practical applications. 


\section{REFERENCES}

[1] Elizabeth Woyke, The Smartphone: Anatomy of an Industry, New Press, 2011.

[2] Electro-Craft Corporation, DC Motors, Speed Controls, Servo Systems: An Engineering Handbook, Elsevier Science \& Technology, 1977.

[3] Kevin Townsend, Getting Started with Bluetooth Low Energy: Tools and Techniques for Low-Power Networking, O'Reilly Media, Inc., 2014.

[4] John Boxall, Arduino Workshop, No Strach press, 2013.

[5] Jeremy Blum, Exploring Arduino: Tools and Techniques for Engineering Wizardry, John Wiley \& Sons, 2013.

[6] Joshua Bloch, Effective Java, Addison-Wesley Professional, 2017. 